TM-1612

\title{
An Algorithm to Calculate the Beam Momentum Distribution from Flying Wire Profiles
}

\author{
X. Q. Wang \\ Fermi National Accelerator Laboratory \\ P.O. Box 500, Batavia, Illinois
}

July 27,1989 


\title{
An Algorithm to Calculate the Beam Momentum Distribution from Flying Wire Profiles
}

\author{
X. Q. Wang
}

July 27,1989

\section{Introduction}

Horizontal flying wire measurements give beam profiles from which information about the beam momentum distribution and betatron distribution can be extracted. When calculating these beam characteristics in the past, for the matter of simplicity, the beam has been assumed Gaussian. For beam profiles which may not be Gaussian, an algorithm to obtain the general beam momentum distribution is developed using the Fourier transform to the beam profiles. Since the profile is the convolution of the momentum distribution and the betatron distribution, using a Fourier transform method makes calculations easier.

\section{Algorithm}

The horizontal beam profiles $d(x)$ can be expressed as the convolution of the momentum distribution function $f\left(\frac{\Delta P}{P}\right)$ and the betatron distribution function $g(x)$ :

$$
d_{1}(x)=\int_{-\infty}^{\infty} f(\tau) g_{1}\left(x-\eta_{1} \tau\right) d \tau
$$

and

$$
d_{2}(x)=\int_{-\infty}^{\infty} f(\tau) g_{2}\left(x-\eta_{2} \tau\right) d \tau
$$

where $\tau \equiv \frac{\Delta P}{P}$ and $\eta$ is the dispersion. The momentum distribution is assumed to be normalized to unity for convenience, and $\eta_{2}$ is assumed to be not equal to 
zero (it is just a matter of choosing the index).

If we assume steady-state beam conditions, the two betatron distribution functions are related to each other as

$$
g_{2}\left(x_{\beta}\right)=\sqrt{\frac{\beta_{1}}{\beta_{2}}} \cdot g_{1}\left(\sqrt{\frac{\beta_{1}}{\beta_{2}}} x_{\beta}\right),
$$

where $x_{\beta}=x-\eta \tau$ is the betatron coordinate.

In order to find the momentum distribution, we take the Fourier transform of the above equations and get

$$
\begin{aligned}
& D_{1}\left(\sqrt{\frac{\beta_{2}}{\beta_{1}}} \omega\right)=F\left(\eta_{1} \sqrt{\frac{\beta_{2}}{\beta_{1}}} \omega\right) \cdot G_{1}\left(\sqrt{\frac{\beta_{2}}{\beta_{1}}} \omega\right), \\
& D_{2}(\omega)=F\left(\eta_{2} \omega\right) \cdot G_{2}(\omega), \\
& G_{2}(\omega)=G_{1}\left(\sqrt{\frac{\beta_{2}}{\beta_{1}}} \omega\right), \\
& F(0)=1 \quad \text { (from the normalization). }
\end{aligned}
$$

Thus we get a relationship between the Fourier transform of the momentum distribution and that of the beam profile, where

$$
F\left(\eta_{2} \omega\right)=\frac{D_{2}(\omega)}{D_{1}\left(\sqrt{\frac{\beta_{2}}{\beta_{1}}} \omega\right)} \cdot F\left(\eta_{1} \sqrt{\frac{\beta_{2}}{\beta_{1}}} \omega\right) .
$$

In a special case of $\eta_{1}=0$, i.e., profile 1 is obtained at the zero dispersion region, the Fourier transform of the momentum distribution is simply

$$
F\left(\eta_{2} \omega\right)=\frac{D_{2}(\omega)}{D_{1}\left(\sqrt{\frac{\beta_{2}}{\beta_{1}}} \omega\right)} .
$$

Since the total number of beam particles is the same for both profiles, we have $D_{1}(0)=D_{2}(0)$. So it is guaranteed in the above expression that $F(0)=1$.

In the case of $\eta_{1} \neq 0$, we can always arrange the indices in such a way that

$$
|\xi|=\left|\frac{\eta_{1}}{\eta_{2}} \sqrt{\frac{\beta_{2}}{\beta_{1}}}\right|<1
$$


(if $|\xi|=1$, the two profiles obtained are actually identical and no further information about any distribution can be extracted from them). Then by applying the relation (5) to its right hand side repeatedly for $N-1$ times, we get

$$
F\left(\eta_{2} \omega\right)=\left\{\prod_{n=0}^{N-1} \frac{D_{2}\left(\xi^{n} \omega\right)}{D_{1}\left(\xi^{n} \sqrt{\frac{\beta_{2}}{\beta_{1}}} \omega\right)}\right\} \cdot F\left(\xi^{N} \eta_{2} \omega\right) .
$$

For any given value of $\omega$, we have

$$
\lim _{N \rightarrow \infty} \xi^{N} \eta_{2} \omega=0
$$

therefore the Fourier transform of the momentum distribution can be expressed as

$$
F\left(\eta_{2} \omega\right)=\prod_{n=0}^{\infty} \frac{D_{2}\left(\xi^{n} \omega\right)}{D_{1}\left(\xi^{n} \sqrt{\frac{\beta_{2}}{\beta_{1}}} \omega\right)}
$$

In actual computation, for any given $\omega$, one can always find an integer $N_{0}$ such that $\xi^{N_{0}} \eta_{2} \omega$ is sufficiently close to zero if $N_{0}$ is sufficiently large. Then Eq. (10) can be approximated by

$$
F\left(\eta_{2} \omega\right) \approx \prod_{n=0}^{N_{0}} \frac{D_{2}\left(\xi^{n} \omega\right)}{D_{1}\left(\xi^{n} \sqrt{\frac{\beta_{2}}{\beta_{1}} \omega}\right)} .
$$

Choice of $N_{0}$ depends on the desired accuracy.

Finally the momentum distribution function is obtained by taking the inverse Fourier transform of $F\left(\eta_{2} \omega\right)$,

$$
f(\tau)=\frac{1}{2 \pi} \int_{-\infty}^{\infty} F\left(\eta_{2} \omega\right) e^{i \eta_{2} \omega \tau} d\left(\eta_{2} \omega\right)
$$

\section{Test of the Algorithm}

Gaussian beam profiles are used to test the program with the above algorithm because in that case the momentum distribution is also Gaussian and its parameters can be calculated analytically. The more general case in which neither $\eta_{1}$ 
nor $\eta_{2}$ is equal to zero is tested. And lattice parameters at the places of the flying wires are chosen to be those for the Fermilab TEVATRON,

$$
\begin{aligned}
& \beta_{1}=160.8 \mathrm{~m}, \\
& \eta_{1}=0.575 \mathrm{~m}, \\
& \beta_{2}=185.85 \mathrm{~m}, \\
& \eta_{2}=6.972 \mathrm{~m} .
\end{aligned}
$$

And two Gaussian profiles are generated with

$$
\begin{aligned}
& \sigma_{1}=\sigma\left(d_{1}\right)=8.121 \times 10^{-4} \mathrm{~m}, \\
& \sigma_{2}=\sigma\left(d_{2}\right)=1.269 \times 10^{-3} \mathrm{~m} .
\end{aligned}
$$

From the above, the calculated $\sigma$ for $\frac{\Delta P}{P}$ is

$$
\sigma_{\frac{\Delta P}{P}}=1.33 \times 10^{-4} \text {. }
$$

In the program, the number of data points is $M=192$ in region $\left(-3 \sigma_{2}, 3 \sigma_{2}\right)$. Figure 1 and 2 give the beam profiles. When using a computer to carry out the calculation, the discrete Fourier transform is used and it must be "good approximation" of the continuous Fourier transform. This results in a cut-off frequency, up to which the discrete Fourier transform is made, which is determined by the spatial separation of two adjacent profile data points (sampling theorem). In addition, for a Gaussian distribution of width $\sigma$, a frequency spectrum up to $\omega_{c}=\frac{3}{\sigma}$ will give satisfactory reproduction of the original profile. For other types of distribution not much different from Gaussian type, the highest frequency component required for a true reproduction of the profile will be close to $\omega_{c}$. In practice, the highest frequency component required for a true reproduction, which is $\sim \frac{3}{\sigma}$, is lower than that restricted by the sampling theorem. Thus the former can be used as the cut-off frequency. In the case that profiles are far from Gaussian, one has to choose the cut-off frequency properly so that their frequency 
spectra contain all the information one wants to know about the momentum distribution.

Figure 3 shows the momentum distribution, obtained from the two given Gaussian bearn profiles, with the width

$$
\sigma_{\frac{A P}{P}}=1.34 \times 10^{-4}
$$

which is in good agreement with the analytical result.

The algorithm has also been tested with real beam profile data for one of the TEVATRON proton bunches. The lattice parameters are the same as given in (13). The bunch profiles are shown in Figures 4 and 5. Gaussian fitting to those profiles gives the beam widths of the same values as in (14). The Fourier transform method gives the bunch momentum profile shown in Figure 6. The width of that profile is

$$
\sigma_{\frac{\Delta P}{P}}=1.33 \times 10^{-4}
$$

The momentum distribution curve is a smooth one because the noise in the flying wire profiles has been filtered when the Fourier transform is taken with a proper cut-off frequency. With that cut-off frequency only the slow-varying part of the profiles, in which one is mostly interested, is preserved. The width change of the momentum profile due to the frequency cut is proven to be negligible from the above test.

\section{Acknowlegement}

I would like to thank Gerry Jackson for supervising this work, and S. Y. Hsueh for useful discussions. 


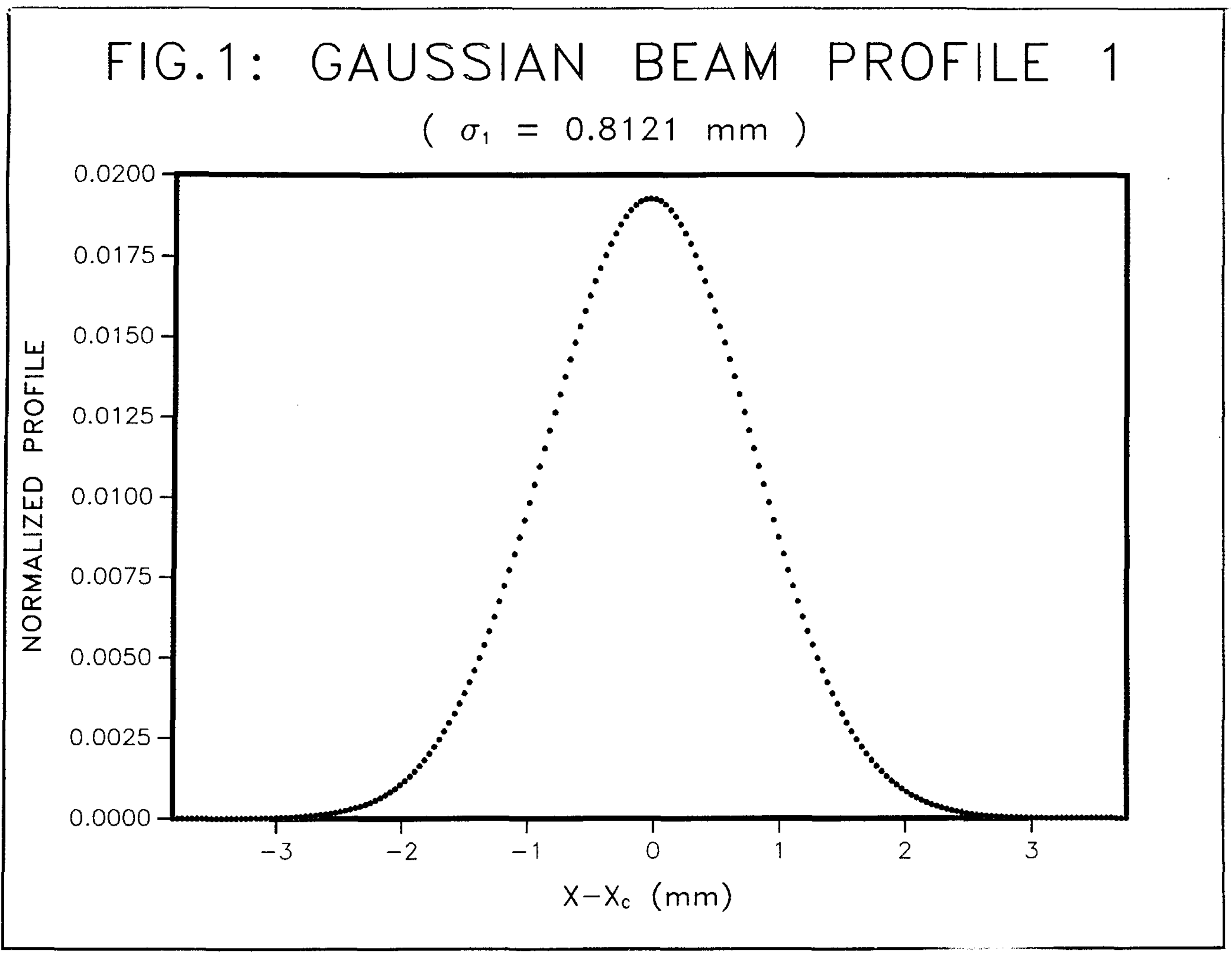




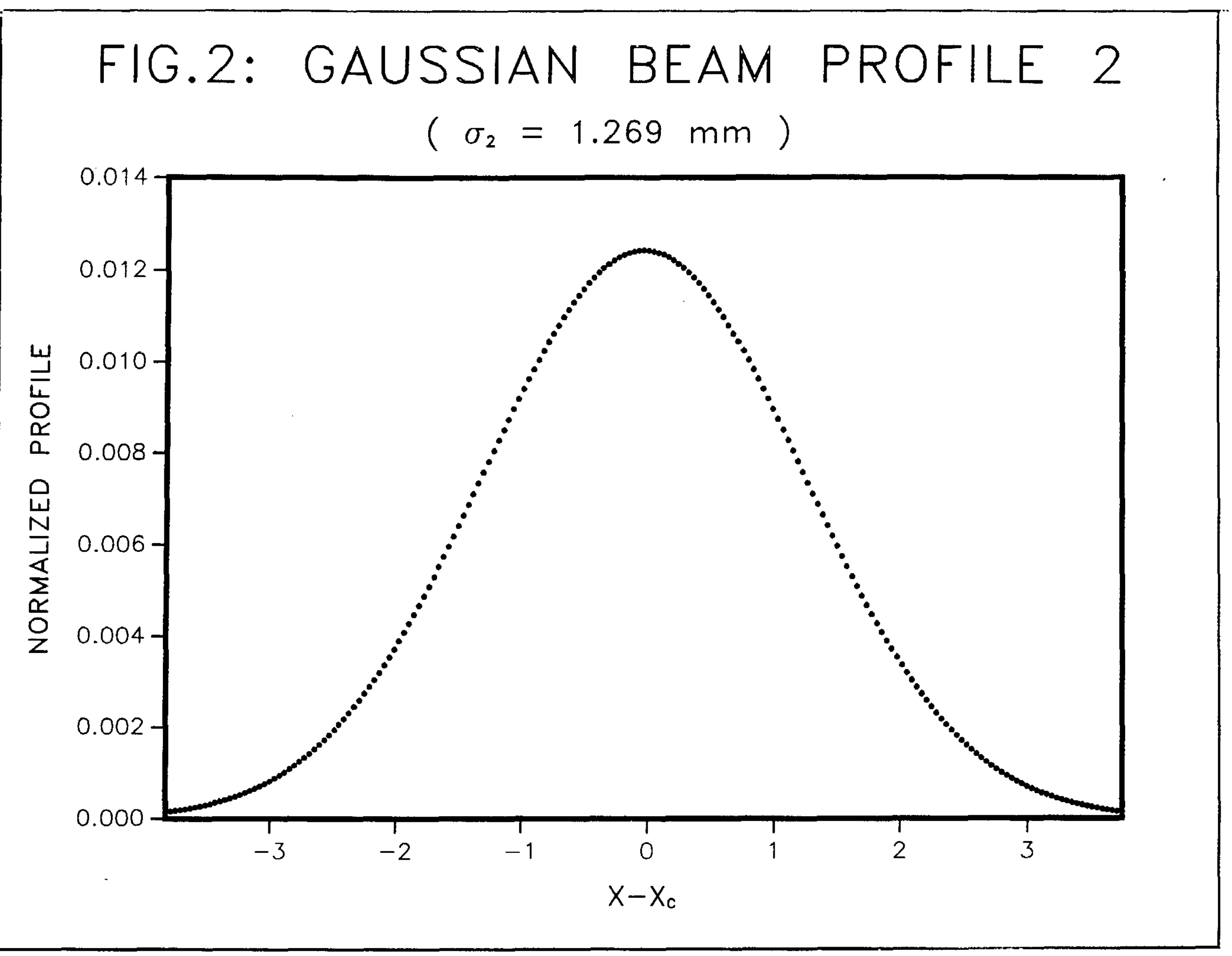


FIG.3: BEAM MOMENTUM PROFILE

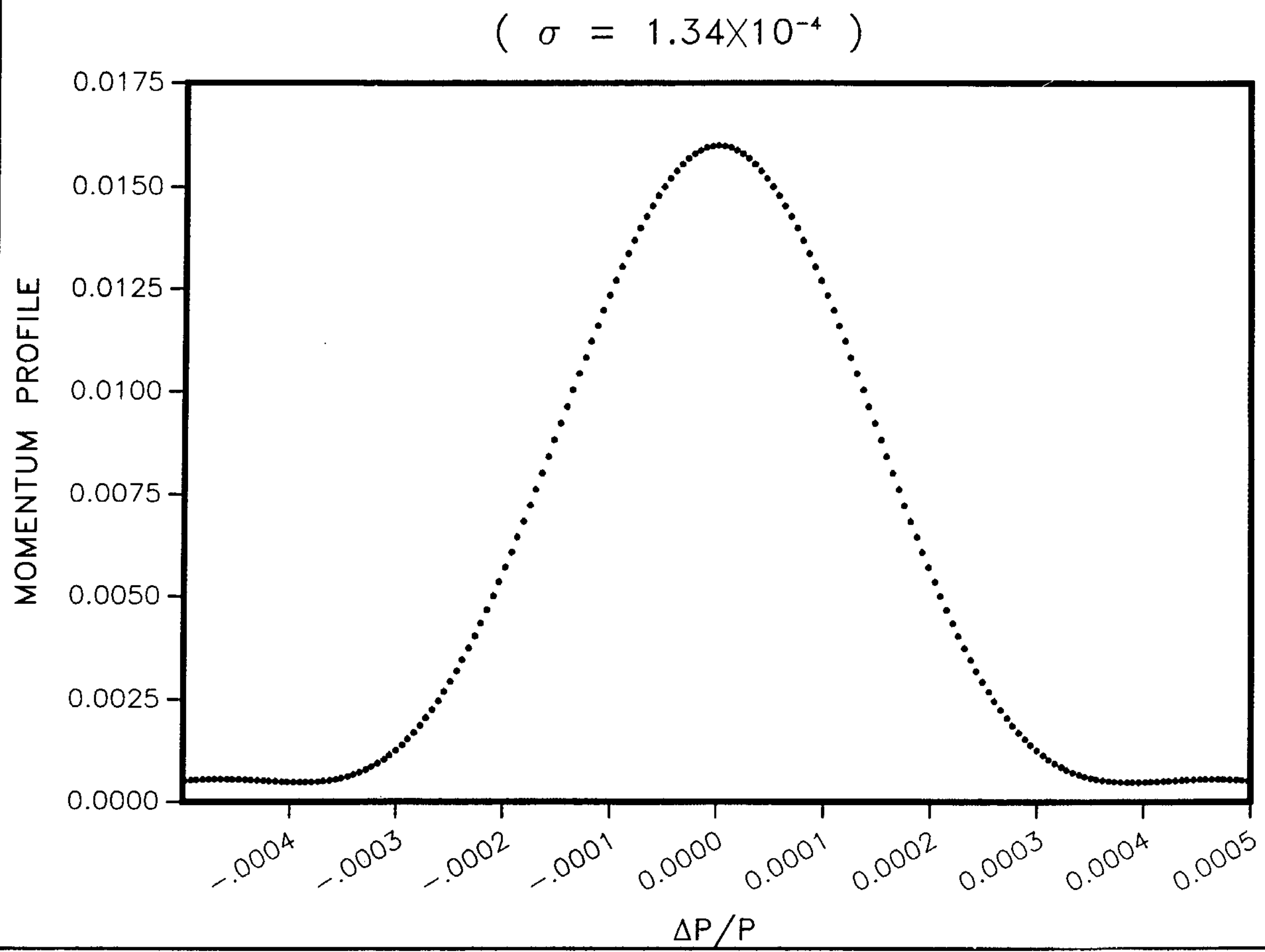


FIG.4: TEV C48 P1 HORZ. PROFILE $\left(\begin{array}{ll}4 / 13 / 89 & 14: 00: 31\end{array}\right)$

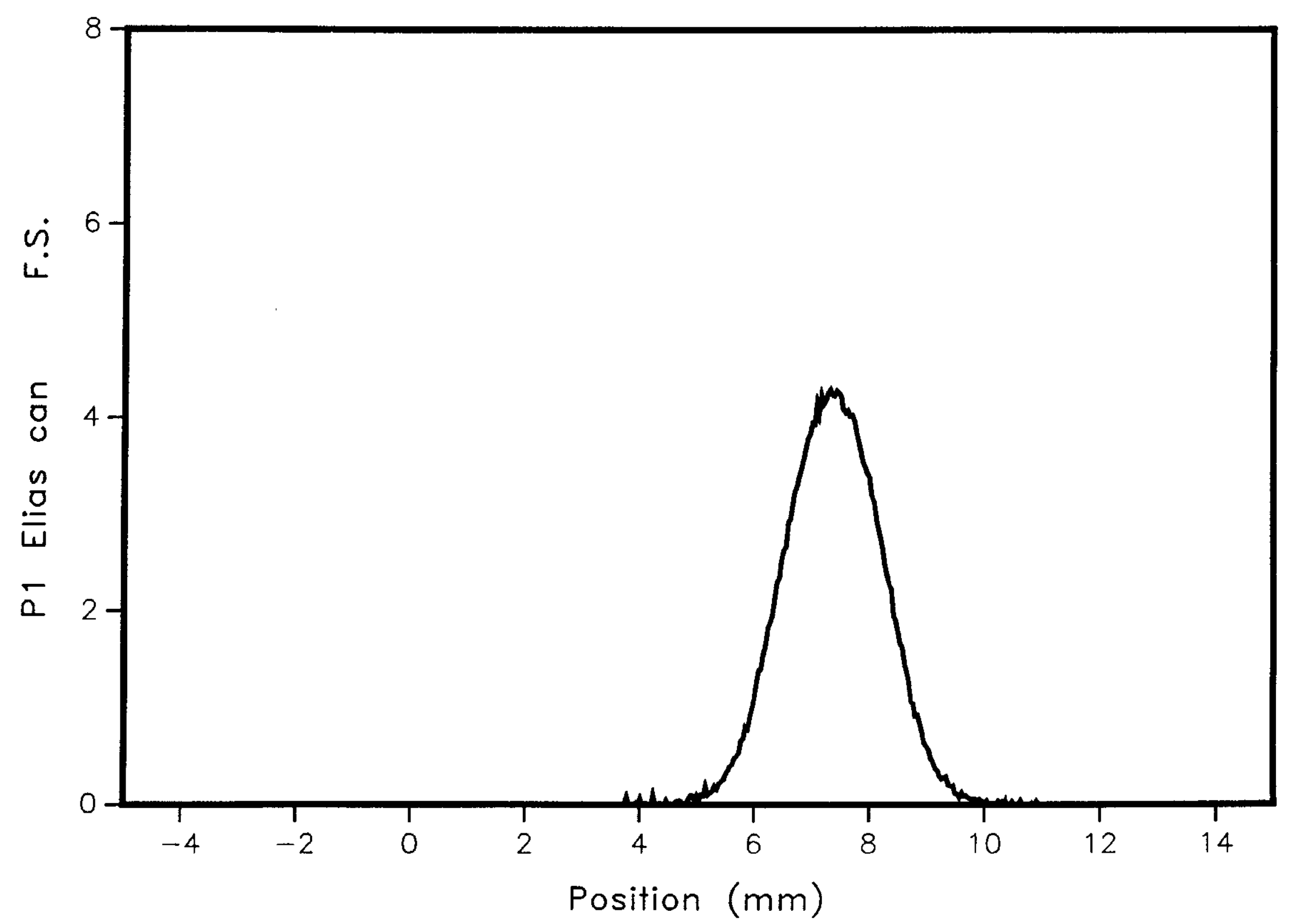


FIG.5: TEV A17 P1 HORZ. PROFILE $(4 / 13 / 89$ 14:00:31)

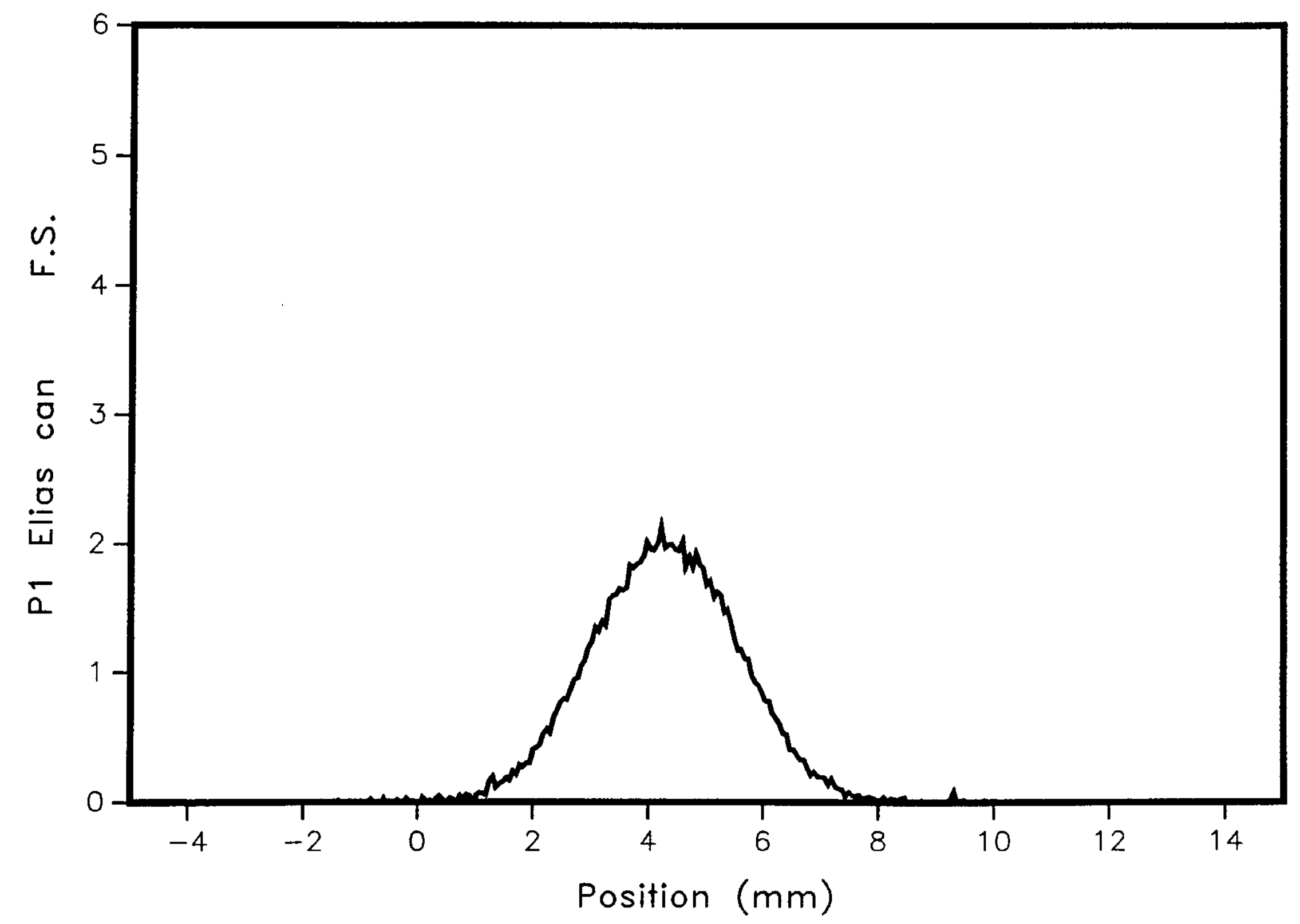


FIG.6: TEV P1 MOMENTUM PROFILE ( 4/13/89 14:00:31)

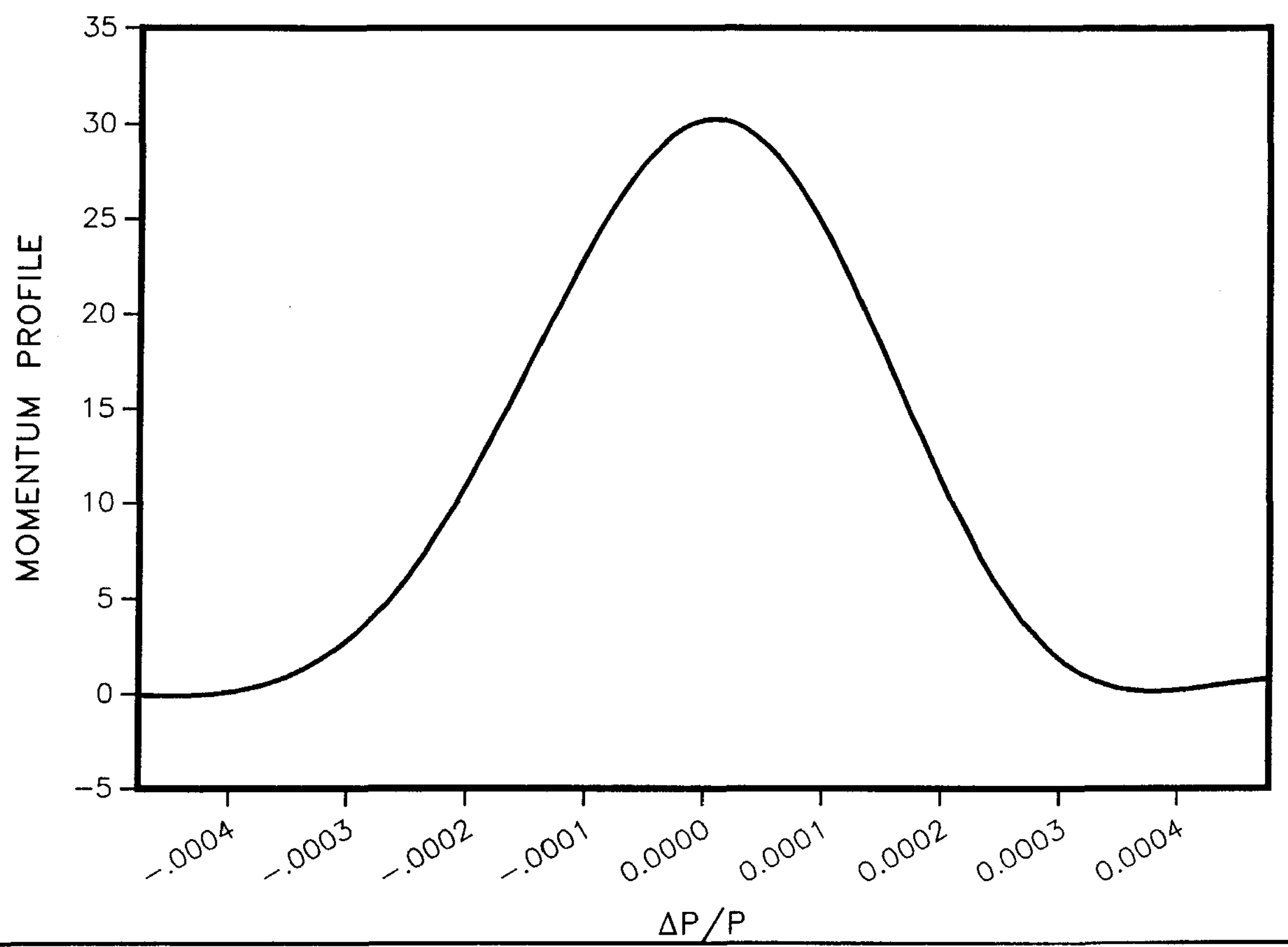

